Felice Jaqueline Schnack ${ }^{1}$

Lidiani de Medeiros Fontana 1

Paulo Roberto Barbosa 1

Loraine Storch Meyer da Silva 1

Clair Maria Martinello Baillargeon 1

Tatiana Barichello 1

Marinete Marins Póvoa 2

Carlos Eugênio Cavasini 3

Ricardo Luiz Dantas Machado 1,3

\section{Enteropatógenos associados com diarréia infantil ( $<5$ anos de idade) em amostra da população da área metropolitana de Criciúma, Santa Catarina, Brasil}

\author{
Enteropathogens associated with diarrheal \\ disease in infants ( $<5$ years old) in a population \\ sample in Greater Metropolitan Criciúma, \\ Santa Catarina State, Brazil
}

\footnotetext{
1 Departamentos de Farmácia, Medicina e Ciências Biológicas, Universidade do Extremo Sul Catarinense. Av. Universitário 1165 , Criciúma, SC 88090-000, Brasil. 2 Seção de Parasitologia, Instituto Evandro Chagas. Av. Almirante Barroso 492, Belém, PA 66090-000, Brasil.

3 Departamento de Doenças Dermatológicas, Infecciosas e Parasitárias, Faculdade de Medicina de São José do Rio Preto.

Av. Brigadeiro Faria Lima 5416, São José do Rio Preto, SP 15090-000, Brasil.

ricardomachado@famerp.br
}

\begin{abstract}
Enteropathogens were investigated in 94 children with diarrhea and 45 age-matched controls, 0 to 5 years old, attending an outpatient unit in Criciúma, Santa Catarina State, Brazil. Cryptosporidium (85.1\%) topped the list of parasite isolates, followed by Entamoeba histolytica (56.4\%) and Giardia lamblia (4.3\%). Four samples contained enteropathogenic Escherichia coli (4.3\%). Samonella and Shiguella were not detected. Only one sample contained rotavirus (1.1\%). Key words Diarrhea; Parasites; Intestinal Diseases; Child Health

Resumo Foi investigada a presença de enteropatógenos em 94 casos de diarréia e 45 casos-controle em crianças de 0 a 5 anos de idade, atendidas no Centro de Saúde Municipal de Criciúma, Santa Catarina. Entre os parasitos isolados, o Cryptosporidium $(85,1 \%)$ foi o mais freqüente, seguido pela Entamoeba histolytica (56,4\%) e a Giardia lamblia (4,3\%). Quatro amostras apresentaram Escherichia coli enteropatogênica (4,3\%). A Samonella e a Shiguella não foram detectadas em nenhuma amostra. Somente um caso foi positivo para o rotavírus $(1,1 \%)$.

Palavras-chave Diarréia; Parasitos; Enteropatias; Saúde Infantil
\end{abstract}


Em populações infantis expostas a altos riscos de aquisição de infecções intestinais, a diarréia se torna o segundo motivo de consultas em ambulatórios médicos. No Brasil, a maior casuística ocorre na região nordeste (Lima et al., 2000). Embora muitos enteropatógenos sejam incriminados na diarréia, o rotavírus tem sido reconhecido como a sua principal causa em crianças em todo o mundo. Algumas bactérias têm sido responsáveis por gastroenterites (El-Sheikh \& El-Assouli, 2001) e, agentes parasitários também têm sido associados a esta nosologia (Chunge et al., 1991). A diversidade geográfica, climática, econômica e social do Brasil pode refletirse na variedade de enteropatógenos nas diferentes áreas endêmicas. Este trabalho objetiva estudar a etiologia da diarréia infantil em amostra da população da área metropolitana de Criciúma, Santa Catarina.

Foram analisadas 94 amostras fecais de crianças (< 5 anos de idade), portadoras de diarréia (menos de sete dias), sem tratamento prévio e 45 amostras fecais de crianças de mesma faixa etária não portadoras de diarréia, atendidas no Centro de Saúde Municipal de Saúde de Criciúma (fevereiro a maio de 2002). Todas as amostras de fezes foram coletadas, transportadas em gelo e processadas dentro de quatro horas após a coleta. Uma alíquota de cada espécime fecal foi congelada para a pesquisa de rotavírus, Giardia lamblia, Entamoeba histolytica e Cryptosporidium. A pesquisa do coproantígeno dos protozoários foi efetuada pelo teste de ELISA (Alexon, Inc., BIOBRÁS). Já para o rotavírus foi realizado o teste de aglutinação (Biolab Merièux). Culturas bacterianas foram feitas para Salmonella, Shiguella e Escherichia coli em meio de MacConkey's, EMB e SS. As culturas com colônias sugestivas foram caracteriza- das por análise bioquímica e sorológica (PROBAC). Para a significância estatística recorreuse ao teste do $\chi^{2}$ (Epi Info 6.0). O nível de segurança para inferência estatística foi de $5 \%$.

Um total de $95,7 \%$ das amostras fecais diarréicas foi positiva para um ou mais enteropatógenos. Foram encontradas infecções mistas em $53 \%$ dos casos de diarréia. A freqüência dos enteropatógenos nas amostras diarréicas está sumariada na Tabela 1. Nas 45 amostras não diarréicas nenhuma bactéria e rotavírus foram detectados, enquanto foi observado positividade de $2,9 \%$ para a $G$. lamblia e $2,5 \%$ para a $E$. histolytica.

A utilização de teste imunoenzimático para pesquisa de coproantígenos é indicada em estudos epidemiológicos para o diagnóstico coletivo ou de populações e em casos isolados com clínica sugestiva e as metodologias microscópicas são inconclusivas (Chapman et al., 1990; Machado et al., 2001; Póvoa et al., 2000). O Cryptosporidium é a causa mais comum de diarréia entre as crianças admitidas, mesmo em associação com outros enteropatógenos. Estes resultados demonstram a importância da investigação de rotina deste parasito. A prevalência da E. histolytica varia em diferentes localidades (Khan et al., 1986; Ruiz-Pelaz \& Mattar, 1999), sendo incriminada também em processos diarréicos infantis. Neste estudo, foi a segunda causa de diarréia, sempre associada com outros enteropatógenos. A Giardia lamblia tem sido associada com episódios diarréicos em inquéritos epidemiológicos, apesar de não ser uma importante causa de diarréia em crianças (Newman et al., 2001). Na população amostral avaliada, a freqüência encontrada não permite incriminá-la como um agente de significante expressão para diarréias entre as cri-

Tabela 1

Freqüência de enteropatógenos em 94 crianças (< 5 anos de idade) com diarréia de acordo com a faixa etária, atendidas entre os meses de fevereiro a maio de 2002, no Centro de Saúde de Criciúma, Santa Catarina.

\begin{tabular}{|c|c|c|c|c|c|c|c|c|}
\hline $\begin{array}{l}\text { Faixa etária } \\
\text { (meses) }\end{array}$ & $\mathrm{n}$ & $\begin{array}{c}\text { Cryptosporidium } \\
\text { n(\%) }\end{array}$ & $\begin{array}{c}\text { Entamoeba } \\
\text { histolytica } \\
\mathrm{n}(\%)\end{array}$ & $\begin{array}{c}\text { Giardia lamblia } \\
\text { n (\%) }\end{array}$ & $\begin{array}{l}\text { Rotavírus } \\
\text { n (\%) }\end{array}$ & $\begin{array}{c}\text { Escherichia coli } \\
\text { enteropatogênica } \\
n(\%)\end{array}$ & $\begin{array}{c}\text { Samonella } \\
\text { n (\%) }\end{array}$ & $\begin{array}{c}\text { Shiguella } \\
\text { n (\%) }\end{array}$ \\
\hline $0-8$ & 2 & $2(2,1)$ & $1(1,1)$ & - & - & - & - & - \\
\hline $9-12$ & 10 & $7(7,4)$ & $7(7,4)$ & - & - & $1(1,1)$ & - & - \\
\hline $13-24$ & 17 & $16(17,0)$ & $14(14,9)$ & $1(1,1)$ & $1(1,1)$ & - & - & - \\
\hline $25-36$ & 19 & $15(16,0)$ & $5(5,3)$ & $3(3,2)$ & - & $2(2,1)$ & - & - \\
\hline $37-48$ & 21 & $17(18,1)$ & $11(11,7)$ & - & - & - & - & - \\
\hline $49-60$ & 25 & $23(24,5)$ & $15(15,9)$ & - & - & $1(1,1)$ & - & - \\
\hline Total & 94 & $80(85,1)$ & $53(56,4)$ & $4(4,3)$ & $1(1,1)$ & $4(4,3)$ & - & - \\
\hline
\end{tabular}


anças estudadas. A baixa freqüência desta infecção pode estar relacionada ao fato de termos utilizado uma única amostra fecal, visto que a eliminação das formas parasitárias deste protozoário é intermitente. No entanto, acreditamos que ocorrência deste parasito seja realmente pequena, já que os resultados encontrados no grupo-controle foram próximos e, que este parasito apresentou, também, freqüências similares $(3,4 \%)$ em pacientes não diarréicos em estudo anterior (Patrício et al., 2002).

A Escherichia coli enteropatogênica não tem sido encontrada na maioria dos países industrializados (Prado et al., 1984). No Estado de São Paulo, é o principal agente patogênico observado em crianças (Medeiros et al., 2001); entretanto, algumas evidências sugerem que elas não são muitos freqüentes em pequenas cidades e áreas rurais (Lima et al., 2001).

\section{Agradecimentos}

Os autores agradecem aos pais e responsáveis dos participantes deste estudo e aos funcionários do Laboratório de Análises Clínicas do Centro de Saúde do Município de Criciúma. À Professora Dra. Andréa Regina Rossit pela leitura crítica do manuscrito. Este estudo recebeu apoio financeiro da Body Shop Foundation.
Estudos em diferentes regiões do Brasil mostram variadas taxas relativas à prevalência das diarréias causadas pelo rotavírus entre crianças (Linhares, 2000). Os resultados deste estudo podem estar associados à distribuição sazonal das gastroenterites pelo rotavírus nas regiões Centro-Oeste e Sudeste/Sul brasileiras. A maior incidência deste enteropatógeno ocorre nos meses de maio a setembro, período mais seco destas regiões (Pereira et al., 1993).

Os resultados deste trabalho nos permitem concluir que a alta prevalência de parasitos entre as crianças diarréicas pode ser um significante problema de saúde pública no Município de Criciúma. Novas investigações devem ser realizadas com o objetivo de detectar outros possíveis enteropatógenos e, especialmente, o levantamento de condições ambientais e de fatores de risco associados às diarréias.

\section{Referências}

CHAPMAN, P. A.; RUSH, B. A. \& McLAUCHLIN, J., 1990. An enzyme immunoassay for detecting Cryptosporidium in faecal and environmental samples. Journal of Medical Microbiology, 32:233.

CHUNGE, R. N.; NAGELKERKE, N.; KARUMBA, P. N.; KALELI, N.; WAMWEA, M.; MUTISO, N.; ANDALA, E. O.; GACHOYA, J.; KIARIE, R. \& KINOTI, S. N., 1991. Longitudinal study of young children in Kenya: intestinal parasitic infection with special reference to Giardia lamblia, its prevalence, incidence and duration, and, its association with diarrhoea and with other parasites. Acta Tropica, 50:39-49.

EL-SHEIKH, S. M. \& EL-ASSOULI, S. M., 2001. Prevalence of viral, bacterial and parasitic enteropathogens among young children with acute diarrhoea in Jeddah, Saudi Arabia. Journal of Health, Population, and Nutrition, 19:25-30.

KHAN, M. U.; SHAHIDULLAH, M.; BARUA, D. K. \& BEGUM, T., 1986. Efficacy of periodic deworming in an urban slum population for parasite control. Indian Journal of Medicine Research, 83:82-88.

LINHARES, A. C., 2000. Epidemiologia das infecções por rotavírus no Brasil e os desafios para o seu controle. Cadernos de Saúde Pública, 16:629-646. 
LIMA, A. A. M.; MOORE, S. R.; BARBOSA Jr., M. S.; SOARES, A. M.; SCHLEUPNER, M. A.; NEWMAN, R. D.; SEARS, C. L.; NATARATO, J. P.; FEDORORKO, D. P.; WUHIB, T.; SHORLING, J. B. \& GUERRANT, R. L., 2000. Persistent diarrhea signals a critical period of increased diarrhea burdens and nutritional shortfalls: A prospective cohort study among children in Northeastern Brazil. Journal of Infectious Diseases, 181:1643-1651.

MACHADO, R. L. D.; FIGUEREDO, M. C.; FRADE, A. F; KUDÓ, M. E.; SILVA FILHO, M. G. \& PÓVOA, M. M., 2001. Comparação de quatro métodos laboratoriais para diagnóstico da Giardia lamblia em fezes de crianças residentes em Belém, Pará. Revista da Sociedade Brasileira de Medicina Tropical, 34:91-93.

MEDEIROS, M. I. C.; NEME, S. N.; SILVA, P.; CAPUANO, D. M.; ERRERA, M. C.; FERNANDES, S. A.; VALLE, G. R. \& ÁVILA, F. A., 2001. Etiology of acute diarrhea among children in Ribeirão Preto-SP, Brazil. Revista do Instituto de Medicina Tropical de São Paulo, 43:21-24.

NEWMAN, R. D.; MOORE, S. R.; LIMA, A. A.; NATARO, J. P.; GUERRANT, R. L. \& SEARS, C. L. A., 2001. Longitudinal study of Giardia lamblia infection in northeast Brazilian children. Tropical Medicine \& International Health, 6:624-634.

PATRÍCIO, H. C.; TCHAIK, R. M.; BARBOSA, P. R. \& MACHADO, R. L. D., 2002. Parasitoses intestinais em Criciúma/SC: Maio - novembro/2001. Revista da Sociedade Brasileira de Medicina Tropical, 35(Sup. 1):262.
PEREIRA, H. G.; LINHARES, A. C.; CANDEIAS, J. A. N. \& GLASS, R. I., 1993. National laboratory surveillance of viral agents of gastroenteritis in Brazil. Bulletin of the Pan American Health Organization, 27:224-233.

PÓVOA, M. M.; ARRUDA, J. E. G.; SILVA, M. C. M.; BICHARA, C. N. C.; ESTEVES, P.; GABBAY, Y. B. \& MACHADO, R. L. D., 2000. Diagnóstico de amebíase intestinal utilizando métodos coproscópicos e imunológicos em amostra da área metroplitana de Belém, Pará, Brasil. Cadernos de Saúde Pública, 16:843-846.

PRADO, V. J.; BRAUN, S. J.; BOSH, P. O.; BERCOVICH, M. W.; REYES, T. M. L. M. \& SAVADA, M. T., 1984. Analisis de Escherichia coli enteropatogeno classico (ECEP) como causa endemica de diarreia aguda en ninos chilenos. Revista Chilena de Pediatría, 55:171-175.

RUIZ-PELAEZ, J. G. \& MATTAR, S., 1999. Accuracy of fecal lactoferrin and other stool tests for diagnosis of invasive diarrhea at a Colombian pediatric hospital. Pediatric Infectious Disease Journal, 18: 342-346.

Recebido em 12 de setembro de 2002

Versão final reapresentada em 21 de fevereiro de 2003 Aprovado em 24 de março de 2003 